\title{
Trois études du pouvoir criminel
}

Actes d'autorité et écriture dans le gang Los Ñetas

Three Studies of Criminal Power: Authority Acts and Writing in the Los Netas

Gang

\section{Martin Lamotte}

\section{(2) OpenEdition}

1 Journals

\section{Édition électronique}

URL : https://journals.openedition.org/conflits/20287

DOI : $10.4000 /$ conflits. 20287

ISSN : $1777-5345$

Éditeur :

CECLS - Centre d'études sur les conflits - Liberté et sécurité, L'Harmattan

\section{Édition imprimée}

Date de publication : 10 octobre 2018

Pagination : 99-120

ISBN : 978-2-343-16812-8

ISSN : 1157-996X

Référence électronique

Martin Lamotte, «Trois études du pouvoir criminel », Cultures \& Conflits [En ligne], 110-111 | été/ automne 2018, mis en ligne le 10 octobre 2020, consulté le 08 janvier 2022. URL : http:// journals.openedition.org/conflits/20287; DOI : https://doi.org/10.4000/conflits.20287 


\title{
Trois études du pouvoir criminel
}

\author{
Actes d'autorité et écriture dans le gang Los Ñetas
}

\section{Martin LAMOTTE}

Martin Lamotte est chargé de recherche an CNRS, attaché an laboratoire CITERES-UMR 7324. Après sa thèse en anthropologie sociale à l'EHESS et son $P h D$ en Urban Studies à l'INRS, sur la globalisation du gang Los Netas, il a réalisé une enquête d'ethno-histoire sur la vie de Carlos La Sombra, le héros fondateur du gang, assassiné à Porto Rico. Il travaille actuellement à une ethnographie des ghettos étatsuniens dans de petites villes $d u$ Sud. Dernières publications : "Du Non-Profit an For-Profit. Vers un "floutage" de la notion de public", Lien Social et Politique, $n^{\circ} 76,2016$, pp. 253-271; "The Neta law the Neta World: Ethics and Imaginaries in Circulation between the South Bronx, Barcelona, and Guayaquil ", Current Sociology, 2016, pp. 1-13 ; "La Police, le "gang” et le Non Profit. Logiques de pacification dans un ghetto Nord Américain», L'Homme, $n^{\circ} 219-220$, 2016/3, pp. 149-174.

F n 2010, Ivan Rodriguez, le chef du gang global Los Ñetas, publie depuis la Lrison où il est incarcéré à Porto Rico, une lettre sur les réseaux sociaux annonçant l'exclusion de Bebo, l'un des présidents de section à New York. Le message désigne ce dernier comme persona non grata, ou insecto (insecte), insulte réservée aux membres du grupo 27, le gang de prison responsable de l'assassinat de Carlos La Sombra, fondateur des Netas à Porto Rico, en mars 1981. Cette exclusion témoigne à première vue de l'autorité du chef suprême dans la vie des Netas et de sa capacité de faire parvenir dans d'autres lieux que le sien ses décisions.

L'histoire des Netas débute dans les années 1980 à l'intérieur du système carcéral portoricain. Selon ses membres, Carlos La Sombra est un prisonnier de droit commun qui protège les prisonniers politiques pro-indépendantistes écroués en même temps que lui. Influencés politiquement par ces derniers, les Ñetas se développent alors comme organisation politique carcérale informelle ayant pour objectif de lutter contre les mauvaises conditions d'incarcération. Au début des années 1990, à la suite de grandes vagues d'immigration de 
Portoricains sur la côte est étatsunienne, La Asociación, l'autre nom que les Netas utilisent pour désigner leur groupe, s’implante dans le système pénitentiaire new-yorkais à partir de l'île-prison de Rikers Island, avant d'évoluer dans les rues de la ville. Forts de plusieurs milliers de membres, ils sont engagés dans de nombreuses guerres de territoire avec d'autres gangs, notamment les Latin Kings. À cette période, les Ñetas sont impliqués dans le trafic de drogue ou d'armes à feu, une de leurs principales activités lucratives. Dès 1993-1994, le groupe s'étend à la côte ouest de l'Amérique latine, par le truchement de deux Ñetas équatoriens, prisonniers à Rikers Island puis expulsés à Guayaquil. La Asociación se développe alors au Pérou et en Bolivie depuis l'Équateur, où elle est fortement implantée et compte plusieurs milliers de membres. Au début des années 2000, les Ñetas s'implantent en Europe, via l'immigration équatorienne en Italie et en Espagne. D'autres gangs, comme les Latin Kings, connaissent la même histoire de circulation et les guerres de gangs se déplacent avec eux ${ }^{1}$.

Le groupe est structuré en Chapters -appelés Capitulos en Espagne et en Amérique Latine ${ }^{2}$ - soit des sections locales autonomes les unes des autres, fortement localisées et dirigées chacune par un président. Dans certains endroits, comme à New York, les Chapters Ñetas sont regroupés dans une Junta Central, une super structure dont le but est de superviser l'ensemble de ces sous-sections. Depuis les années 1990 et la montée en puissance de La Asociación à New York, le monde Netas s'articule autour de deux pôles : Porto Rico, où est emprisonné le líder máximo, soit le chef des Netas, et New York, centre dynamique du mouvement.

Pourtant, l'échelle globale de La Asociación interroge quant à la capacité de la gouverner. Trois questions organiseront la réflexion dans cet article : Comment diriger un monde criminel depuis une prison à Porto Rico ? Comment ordonnancer le quotidien dans un réseau étendu ? Et finalement, comment penser et agir sur l'avenir et le devenir criminel ? Pour répondre à ces questions, je voudrais revenir sur trois logiques d'action qui définissent trois fonctions clefs dans la structure associative des Netas : diriger, pour le líder máximo; ordonnancer, pour le secrétaire du Chapter; et prévoir, pour le président de Chapter.

Je m'intéresse ici à la façon dont le pouvoir s'exerce au sein de La Asociación en décrivant trois logiques d'action dans des situations particulières et la façon dont celles-ci reconfigurent, ou non, les relations de pouvoir dans le Monde Netas à un moment précis. En ce sens, je ne propose pas une

1. Brotherton D. et Barrios L., The Almighty Latin King and Queen Nation Street Politics and the Transformation of a New York City Gang, New York, Columbia University Press, 2004, p. 464.

2. Par souci de précision, j'utiliserai dans le reste de ce texte le terme de Chapter pour décrire les sections aux États-Unis et Capitulo pour décrire celles en Amérique Latine ou en Espagne. 
théorie du gouvernement criminel, mais j'essaie d'examiner, dans une situation donnée, les logiques d'action, les outils et la façon dont les individus agissent pour gouverner. Je m'inscris dans une approche d'anthropologie politique telle que Michel Naepels la définit, opérant un « déplacement pragmatique $^{3}$ » depuis l'étude des typologies ou procédures institutionnelles à l'analyse des capacités mises en œuvre dans l'action ou dans la pratique. Enfin, ce texte doit beaucoup à ma lecture croisée de l'ouvrage Le siècle des chefs d'Yves Cohen ${ }^{4}$, qui m'a permis de penser la place du chef au sein de La Asociación et surtout les diverses logiques d'action qui fondent son autorité, ainsi qu'aux travaux de Déborah Puccio Den sur l'usage de l'écrit qu'en font les membres de la mafia Cosa Nostra ${ }^{5}$.

Afin d'analyser les différents actes d'autorité attachés à ces trois logiques d'action, je voudrais explorer la place centrale de l'écrit dans le Monde Ñetas. Ainsi, pour diriger, le líder máximo utilise les lettres ; pour ordonnancer, le secrétaire archive les «messages de conscience » (les lettres personnelles envoyées par les membres); pour prévoir, le président utilise le plan. Si, à la fin des années 1980, ces écrits avaient pour support le papier, ils circulent aujourd'hui sur les réseaux sociaux, renforçant le caractère global de $\mathrm{La}$ Asociación.

De ces trois types d'écrits émergent des types d'autorités particulières que je voudrais décrire. La façon dont ces trois acteurs s'en emparent permet de définir les trois logiques d'action au cour de mon analyse et de dessiner une pragmatique du pouvoir criminel.

Lorsque je rencontre Bebo en 2011, il a été successivement secrétaire et président de la Junta Central de New York, ainsi que porte-parole du líder máximo avant d'être déchu de toutes ces fonctions. Pendant deux ans, nous avons réalisé une série d'entretiens d'histoire de vie. C'est à travers lui que j'ai rencontré ses anciens Hermanitos - les frères Netas - à New York, à partir de l'automne 2011. J'ai ainsi participé aux activités des membres, que ce soient les cérémonies spirituelles honorant la mort de Carlos La Sombra, les fêtes, les réunions de Chapters ou les activités publiques du groupe, telles que les manifestations contre la violence policière ou les regroupements funéraires aux coins de rue où des membres ont été tués. C'est aussi grâce à Bebo que j’ai pu poursuivre cette ethnographie en Espagne, où il m'a mis en contact avec le Padrino, un président de Chapter à Barcelone. J'y ai passé deux années, en

3. Naepels M., Ethnographie, pragmatisme, histoire: un parcours de recherche à Houailou (Nouvelle-Calédonie), Paris, Publications de la Sorbonne, 2011, p. 150.

4. Cohen Y., Le siècle des chefs: une histoire transnationale du commandement et de l'autorité (1890-1940), Paris, Éditions Amsterdam, 2013, p. 871.

5. Puccio-Den D. " "Dieu vous bénisse et vous protège”. La correspondance secrète du chef de la mafia sicilienne Bernardo Provenzano (1993-2006) ", Revue de l'bistoire des religions, $\mathrm{n}^{\circ} 228,2014$, pp. 307-326. 
vivant par périodes dans l'appartement squatté par le Padrino et sa compagne de l'époque. Enfin, à travers les contacts du Padrino, je me suis rendu en 2014 à Guayaquil pour un court terrain de recherche où j'ai rencontré Daniel, le secrétaire d'un Capitulo important dans la ville, berceau de l'implantation Netas en Equateur.

À Barcelone, j’ai suivi les activités du Capitulo du Padrino où j'ai été initié, ce qui m’a permis d'en devenir le secrétaire pendant une année. J'ai ainsi pu prendre part de manière plus importante aux activités Ñetas, comme les réunions, qui m'étaient auparavant interdites. Ma position de chercheur a toujours été clairement annoncée au début de toute rencontre, mais elle fut rapidement oubliée, il me semble, ou passée en arrière-plan, lors de ces interactions. Pour autant, cette intégration fut lente et parfois difficile. Par exemple, ce n'est qu'à Barcelone que j'ai pu me rendre compte de l'importance de l'écrit au sein des Ñetas. À New York, au contraire, la méfiance vis-à-vis de ma présence et mon amitié avec Bebo, encore considéré par certains membres comme un insecto, ne me permit pas d'y tisser des liens aussi forts qu'à Barcelone ou Guayaquil.

Ainsi, mon approche a-t-elle créé certains biais. Par exemple, si mes séjours répétés et prolongés dans la maison du Padrino à Barcelone m’ont permis de développer une forte amitié, j'ai dans le même temps été associé au président qu'il représentait pour nombre d'autres Netas. J'ai essayé de nuancer cette posture en passant le plus de temps possible avec les Netas de sa section, ainsi qu'avec d'autres Netas à Barcelone, mais il semble qu'en Espagne je reste associé au Padrino, du moins à Barcelone. Durant quatre années, j'ai mené une ethnographie multi-située ${ }^{6}$, me déplaçant dans le Monde Netas afin de comprendre comment se structure cette communauté globale. Ce déplacement sur l'échelle géographique s'est accompagné d'un déplacement sur l'échelle temporelle puisque les Netas ont cette particularité d'être un gang qui existe depuis les années 1970. Ils contredisent ainsi l'une des premières définitions du phénomène de gang énoncée par Trasher en 1927, qui voit dans les gangs un épiphénomène ${ }^{7}$. L'histoire, comme nous le verrons par la suite, est centrale pour les membres de La Asociación, qui ont écrit leur récit dans un livre appelé le Liderato. J'ai, donc, à la fois enquêté sur le quotidien des Netas et sur leur histoire, ou plutôt sur leur façon d'écrire cette histoire.

En 2011, Bebo, le Padrino et Daniel ont entre 30 et 40 ans. Ils sont considérés, par leur Hermanitos qui ont pour la plupart entre 18 et 25 ans, comme des « Original Gangsters », de vieux Netas, reconnus pour leur sagesse et leur

6. Marcus G. E., «Ethnography In/Of the World System: The Emergence of Multi-Sited Ethnography », Annual Review of Anthropology, vol. 24, n 1, 1995, pp. 95-117.

7. Trasher F. M., The Gang: a Study of 1313 Gangs in Chicago, Chicago, University of Chicago Press, (1927) 1968. 
passé prestigieux. Mon ethnographie du monde Netas n'est pas uniquement liée à ces trois personnages clefs, mais cet article repose en grande partie sur ces trois figures qui me semblent emblématiques de l'organisation et des rapports de force structurant La Asociación. Enfin, je tiens à préciser que la question du pouvoir n'est pas plus centrale chez les Netas qu'elle peut l'être dans d'autres groupes. Il serait ainsi erroné de donner l'impression que la vie dans La Asociación est orchestrée uniquement par des jeux de pouvoir soldés par la violence, comme ce peut être le cas dans les versions romancées que l'on retrouve dans la trilogie Le Parrain ou la série Gomorra. Bien que ce ne soit pas le propos de cet article, les Netas sont surtout concernés par leur devenir, leur philosophie de vie et les pratiques de transformation de soi qu'ils mettent en place au quotidien ${ }^{8}$.

\section{Diriger : Distance et présence épistolaire du lider máximo}

\section{L'exclusion de Bebo}

La procédure d'exclusion de La Asociación, telle que celle qu'a subie Bebo, est peu courante, et celle-ci doit être rendue publique à l'ensemble du Monde Netas. À partir des années 2000, les Netas se servent de plusieurs réseaux sociaux sur lesquels ils publient ce genre d'informations. Ainsi, un Ñetas en Espagne ou en Équateur peut être au courant des affaires d'expulsions dans le New Jersey. Une pareille sentence doit, selon le code de règles Netas, être avalisée par une autorité supérieure, qu'il s'agisse d'une Junta si la décision est prise au niveau du Chapter ou du líder máximo lui-même, pour les cas les plus graves. Une fois l'expulsion rendue publique, tous les Ñetas sont censés couper tout contact avec l'insecto. Au-delà de la violence symbolique d'une telle procédure, dans la situation de paupérisation avancée qui touche la plupart des membres, se voir ainsi couper de ses liens avec le gang peut engendrer une grande insécurité et instabilité économique. En effet, $\mathrm{La}$ Asociación permet, entre autres, l'établissement de liens et de réseaux d'entraide suffisamment puissants pour aider un membre dans le besoin. Enfin, être déclaré insecto peut engendrer des types de violence physique. L'insecto peut alors être agressé par les membres de son ancien Chapter, comme c'est le cas de Bebo, qui fait face à des menaces de mort émises par ses anciens amis. Mais il se trouve aussi dépourvu de la protection offerte par le gang et sans support face à la violence d'autres gangs.

Bebo reste peu prolixe sur les raisons de sa mise à l'écart. Son expulsion sera par ailleurs révisée en 2013, lors d'un changement de líder máximo, et Bebo redeviendra pour un temps un membre à part entière. Il perd cependant ses fonctions de président et de porte-parole.

8. Lamotte M., « The Neta law the Neta World : Ethics and Imaginaries in Circulation between the South Bronx, Barcelona, and Guayaquil », Current Sociology, 2016, pp. 1-13. 
Chaque Chapter doit, selon l'organigramme Netas, être composé d'un président (le primero), d'un secrétaire et d'un chef de la discipline (le moderador de disciplina). En fonction de l'importance numérique du Chapter, s'ajoutent à ces fonctions celles de vice-président (le Segundo) et d'asesor. Celui-ci est un membre plus ancien, réputé pour sa sagesse, qui sera consulté lors de conflits entre les membres du même Chapter ou par le Primero en cas de besoin. Chaque Chapter est dirigé par un président, le primero, élu par les membres. Enfin, il peut y avoir un trésorier et un vocale, qui assure le lien entre le groupe et les autres Chapters, voire la Junta Central si elle existe. Toutes ces positions sont électives, votées par l'ensemble des membres du groupe appelés guerreros. Chaque guerrero a le droit de parole aux réunions collectives et celui de proposer la destitution des responsables élus. Il n'y a pas de discrimination de principe envers les femmes ; plusieurs d'entre elles ont été primero de Chapters importants, dirigeant parfois des Chapters majoritairement masculins. Jusqu'à ce jour, les homosexuels ont le droit d'être membres mais pas celui de voter. Au début des années 1990 à New York, seuls les Netas d'origine portoricaine avaient le droit d'accéder à des positions hiérarchiques.

Cet organigramme est le même dans tous les groupes Netas, que ce soit à New York, en Floride, en Équateur ou à Barcelone. Formellement, le líder máximo dirige ce monde Neta. Il doit être, selon les règles internes, un prisonnier à Porto Rico. Dans le contexte portoricain, les Netas n'existent que dans les prisons et n'ont pas de «Chapters de rue ». Un membre de La Asociación perd ses droits et son appartenance dès lors qu'il sort de prison. Il redeviendra automatiquement membre dans le cas d'une nouvelle incarcération, mais ne retrouvera pas nécessairement son statut hiérarchique.

L'élection du líder máximo reste opaque pour le reste des membres du monde Ñetas qui l'apprennent a posteriori. Ainsi, en 2013, Ivan Rodriguez est transféré de la prison fédérale de Porto Rico à une prison fédérale dans le Colorado, ce qui l'empêche, selon les règles Ñetas, d'occuper la place de líder máximo. Suite à une élection à Porto Rico, il est remplacé par Papi Caro, ce que Bebo, le Padrino ou Daniel apprendront ultérieurement. Cette organisation de la chefferie Ñetas repose sur une forme pyramidale. De ce fait, se pose une question centrale pour le líder máximo : comment gouverner un gang aux ramifications internationales depuis l'espace clos de sa prison et sans la possibilité de circuler ? 


\section{S'adresser au monde}

Le líder máximo peut s'adresser aux Ñetas en dehors de Porto Rico de deux manières : à l'oral, par téléphone, ou à l'écrit, par échange épistolaire. Dans ce dernier cas, les lettres transitent par un intermédiaire extérieur à la prison qui lui apporte directement le courrier. En 2014, Bebo me parle ainsi de la venue de Vera à New York, l'intermédiaire de Papi Caro, nouvellement élu au poste de líder máximo. Vera fait partie du comité de soutien aux familles de prisonniers, une association à but non lucratif qui regroupe les familles des membres Ñetas et milite pour la reconnaissance de leurs droits. Cette organisation permet d'assurer le lien entre les prisons et la vie civile à Porto Rico, mais aussi entre les membres portoricains incarcérés et le reste du Monde Netas. C'est ainsi que Vera est envoyée à New York par Papi Caro afin d'établir un pont avec les Ñetas de la ville américaine. Elle se rend dans le Chapter du New Jersey où Bebo est Asesor. Bien que «blanchi », selon ses dires, par une lettre envoyée par Papi Caro à l'ensemble des Ñetas new-yorkais, Bebo ne veut pas redevenir complétement actif. Il est possible depuis peu de «sortir » du gang en se déclarant comme membre non actif. Auparavant, et selon le dicton Netas, il n'y avait que deux voies de sortie possible : la bible à la main ou dans un sac mortuaire », en devenant prêtre ou une fois mort.

Vera est la porte-parole personnelle de Papi Caro et c'est donc par elle que transitent les lettres qui lui seront dorénavant adressées. Elle s'assure de la bonne application des directives de Papi Caro et fait remonter ses observations au líder máximo. Papi Caro repose ainsi entièrement sur cette intermédiaire, qui détient un grand pouvoir au sein de l'organisation, malgré le fait qu'elle n'ait pas été élue. En 2016, Bebo et El Gordo, le président du Chapter de New Jersey, entrent en conflit avec Vera. Remettant en cause son autorité, ils n'acceptent pas de recevoir d'ordres de sa part. Les deux hommes essaient d'entrer directement en contact avec Papi Caro. Bebo, qui avait jusqu'au début des années 2000 un accès direct aux Netas portoricains, prend contact avec plusieurs membres incarcérés dans la même prison que Papi Caro. Cependant, ses tentatives échouent et Vera fait déclarer El Gordo persona non grata, obligeant ainsi une recomposition interne du Chapter du New Jersey. Lorsque je retrouve Bebo en mai 2018, il me fait part de sa décision de se tenir éloigné de La Asociación. La décision de Papi Caro d'exclure El Gordo et le pouvoir accru de Vera ont mis fin à ses désirs de reconquête. Le Chapter de New York a aussi été démantelé, suite à des conflits avec Vera, et Bebo, la quarantaine, considère qu'il est maintenant trop âgé pour ces luttes intestines.

Les lettres échangées entre prison et calle (les Chapters de rue) sont fréquentes. Elles sont régies par un code particulier, qui change parfois selon les lieux, mais qui a pour finalité de cacher le contenu des messages à la surveillance pénitentiaire. Ainsi, le Padrino à Barcelone ne signe jamais de son nom 
lorsqu'il envoie une lettre en prison. De la même façon, il lui arrive de ne pas expliquer clairement le but de sa démarche. Certains mots sont préférés à d'autres, pour ne pas attirer l'attention des gardiens qui pourraient relire le courrier. Lorsque les lettres sont potentiellement compromettantes, les Ñetas demandent à un prisonnier autorisé à sortir temporairement afin de délivrer le message à l'oral. Cette méthode est, par exemple, utilisée pour avertir les Netas incarcérés de l'arrivée d'un nouveau membre en prison et attester de son appartenance à tel ou tel Chapter de rue. À l'inverse, si l'information à transmettre est considérée peu sensible pour être exprimée publiquement, les Ñetas communiquent par l'intermédiaire de Facebook, whatsapp ou autres. Ces plateformes servent de lieux de débats, mais sont aussi le support de conflits intenses.

La question du commandement de Papi Caro peut être interrogée par le couple distance/présence. En effet, Papi Caro est un chef qui dirige à distance, depuis l'espace de sa cellule, et toute son action vise à réduire cette distance. L'historien Yves Cohen ${ }^{9}$, dans son histoire transnationale du commandement et de l'autorité, distingue l'espace d'action immédiate (ou zone d'activité) et l'espace des effets (ou encore zone d'impact) à propos des techniques de management qui se développent dans les grandes organisations industrielles au XXe siècle. La zone d'activité de Papi Caro est constituée par sa cellule, l'espace de la prison et sa relation avec son intermédiaire, alors que sa zone d'impact couvre l'espace où ses décisions sont appliquées. Or, la position de Papi Caro est paradoxale en ce que sa zone d'activité est particulièrement réduite, alors que sa zone d'impact semble aussi grande que le Monde Ñetas lui-même. Pour cela, le líder máximo doit mettre en place une série de «techniques de la distance 10 » visant à lier sa zone d'activité et sa zone d'impact et à réduire l'écart entre les deux. Grâce aux échanges épistolaires, le líder máximo a su transporter sa présence dans l'ensemble de l'espace Ñetas. Dans un mouvement inverse, l'utilisation d'un intermédiaire se mouvant dans le monde, comme Vera aujourd'hui ou Bebo auparavant, permet de transporter l'espace lointain auprès du líder máximo. Ce système fonctionne de pair avec une «technologie de l'effroi ${ }^{11}$ », qui fait partie de l'outillage de la distance du líder máximo. En effet, les lettres d'exclusion, que ce soit celle de Bebo ou de El Gordo, permettent de rendre à la fois effective la technique de distance du chef portoricain et de s'assurer du maintien de son ordre et autorité. Les lettres du líder máximo sont donc la matérialisation d'un pouvoir de type disciplinaire. Pour autant, celles-ci ne marquent pas, comme c'est le cas de la Cosa Nostra qu'étudie Puccio-Den ${ }^{12}$, un tournant autoritaire de La Asociación, puisque, comme nous le verrons par la suite, la verticalité des échanges avec le líder

9. Cohen Y., op. cit.

10. Ibid., p. 564.

11. Ibid., p. 573.

12. Puccio-Den D., op. cit. 
máximo est nuancée par une horizontalité des échanges entre les Netas - que ce soit entre les présidents pour la définition de plans de travail, ou entre les membres avec l'usage des messages de conscience. De plus, l'autorité du líder máximo n'est pas sans limites ou contraintes et doit faire face à plusieurs contestations. Ainsi, en 2003, Ivan Rodriguez, le líder máximo de l'époque, envoie une lettre aux Ñetas à Barcelone critiquant les violences commises et va jusqu'à leur reprocher de pas être de vrais Netas. Selon le Padrino, cette annonce provoqua une véritable colère à Barcelone et entama le début de relations difficiles entre les Ñetas en Espagne et Ivan Rodriguez. Cela n'empêche pas le Padrino d'entrer en contact avec Papi Caro, dès sa désignation en remplacement d'Ivan Rodriguez, pour rétablir de bonnes relations entre l'Espagne et Porto Rico. De plus, être en contact plus ou moins direct avec le líder máximo est le signe d'une position de prestige, ce que tente d'exploiter le Padrino.

\section{Économie politique du commandement à distance}

L'œil du chef dépend ainsi du bon vouloir, et de la justesse du regard de ses intermédiaires. Incarcéré, le líder máximo est limité dans ses échanges épistolaires, puisque ceux-ci passent par Vera. Comme l'indique Yves Cohen, le commandement à distance « ne repose pas seulement sur les techniques d'information, mais aussi sur une économie politique : qui dispose de quoi ? " 13 . Or, dans ce cas, le chef ne dispose pas de grand-chose, ce qui limite considérablement son pouvoir. Il pourrait alors être avancé que la puissance du chef existe dans une forme de présence sacrée, plus ou moins dépourvue de force effective, mais qui gagnerait en autorité perpétuelle et universelle. Cette hypothèse est étayée par le fait que peu de choses sont connues du líder máximo. Si Papi Caro devient le chef Netas en 2013, il semble que ni Bebo, ni El Gordo, ni même le Padrino n'aient entendu parler de ce dernier avant son élection. Il est vrai que, hormis les procédures d'exclusion ou les visites de son intermédiaire, la place du líder máximo dans le quotidien des vies des membres Netas à Barcelone, New York et Guayaquil semble minime. La distance avec le chef serait ainsi transmuée en rapport avec le sacré. Dans son étude sur les pizzinis, ces lettres que le chef de la Cosa nostra adresse aux membres de la mafia, Deborah Puccio-Den montre la mobilisation d'une stratégie épistolaire pour fabriquer les instruments du pouvoir du chef et exercer une autorité de type spirituel au sein de la Cosa nostra. Cependant, dans le cas des Netas, ce rapport au spirituel ne rend pas compte de la dimension punitive des lettres du líder máximo et de leur conséquence bien réelle en termes d'exclusion. Porto Rico constitue un pôle central dans le Monde Ñetas, et les lettres ont des effets pouvant s'avérer tragiques dans la vie personnelle des membres, ce qu'illustre l'exclusion de Bebo. Le mystère autour du Chef et le manque de transparence

13. Cohen Y., op. cit., p. 571. 
quant à sa désignation rendent sa personne d'autant plus inaccessible que le pouvoir qu'il incarne est déterritorialisé.

L'acte d'écriture du chef portoricain est essentiel à deux titres. D'une part pour le chef lui-même, car son autorité en dépend. D'autre part, pour les membres Netas dans leur ensemble, car il est contingent de l'idée même d'une communauté Ñetas à travers le monde. Le monde Ñetas s'appuie, entre autres, sur l'existence du líder máximo qui en est le pivot et le support. Autrement dit, au-delà de la question de la réalité de son autorité, son existence permet de faire tenir le monde. Cet argument plaide en faveur d'une conception de l'autorité du chef qui soit à la fois sacrée, ou plutôt symbolique, et réelle. Je rejoins ici l'argument d'une pragmatique des actes du chef, avancé par Yves Cohen. Les actes du líder máximo ne font pas seulement ce qu'ils sont censés faire (dans le cadre de cet article : exclure, adouber, communiquer), ils confirment aussi l'autorité, ou du moins l'existence du chef 14 et donc, en miroir, l'existence de ceux qui ont un chef.

\section{Ordonnancer : les archives du secrétaire}

\section{Le secrétaire}

En février 2014, à Barcelone, le Padrino me demande de devenir secrétaire de son groupe. Cela fait maintenant un peu plus de six mois que je participe régulièrement aux réunions du nouveau Capitulo qu'il a créé. Le groupe se réunit régulièrement dans des parcs ou des terrains de sport pour discuter des principes philosophiques de La Asociación, boire des bières et parler de la situation de chacun. Puisque le Padrino interdit aux membres de son Capítulo de vendre de la drogue au nom de La Asociación, ceux qui s'y adonnent le font de manière individuelle en dehors des activités du groupe. Plusieurs fois, le Capitulo organise des réunions avec d'autres groupes Netas de la ville, afin de favoriser la cohésion au sein de La Asociación et mettre en place le plan de réunification mondial du Padrino (voir ci-dessous).

Arrivé d’Équateur à Barcelone au début des années 2000, le Padrino est une figure importante des Netas. Connu pour avoir été un chef de guerre important dans le conflit qui opposait à Guayaquil les Netas et les Latin Kings ${ }^{15}$, il a participé à Barcelone à la première Junta Central organisée en 2006. Bien que celle-ci ait été dissoute suite à des désaccords internes, le Padrino conserve un certain prestige qui lui permet d'être écouté par la plupart des Netas en Espagne. À partir de 2013, après avoir été quelque temps

14. Voir Austin J. L., Quand dire, c'est faire, Paris, Éditions du Seuil, 1970, dans l'ouvrage de Yves Cohen sur une pragmatique des actes du langage qui « disent ce qu'ils disent et en même temps disent qu'ils disent ». Cohen Y., op. cit., p. 622.

15. Tout comme les Netas, les Latin Kings se sont développés en Équateur et en Espagne, réactivant à chaque fois les conflits entre ces deux gangs. 
non-actif, il décide de relancer un Capitulo, avec plusieurs nouveaux membres. Compte tenu de la petite taille de son groupe, les positions à pourvoir sont ouvertes. Puisque je vis en alternance chez lui, et puisque j'ai toujours à portée de main mon carnet de terrain, il semble aller de soi pour les membres du groupe que je m'occupe de la prise de notes. C'est Hector, l'un des membres du Capitulo, élu chef de la discipline, qui propose mon nom et je suis élu par un vote lors d'une réunion de section. Je peux dès lors avoir accès à l'ensemble des papiers circulant de manière interne dans le Capitulo puisqu'il me revient la tâche de les archiver. Cela me donne, par ailleurs, une position et un rôle au sein du Capitulo et du Monde Ñetas, du moins à l'échelle locale barcelonaise.

Ainsi, en plus de participer aux réunions du groupe, le secrétaire doit tenir à jour le cahier du Capitulo. Il y retranscrit chaque procès-verbal des réunions du collectif, qui sera signé par l'ensemble des membres présents. Le secrétaire doit y acter les présences et retards éventuels, les prises de parole et inscrire tout échange d'argent, entrant ou sortant, dans le Fondo, le fond commun servant de banque pour chaque Capitulo. Ce cahier constitue une sorte d'archive du quotidien du groupe. Il est conservé par le secrétaire, puis, une fois rempli, par le président. Contenant un ensemble d'informations précises sur les affaires passées et en cours, ces archives peuvent être mobilisées lors d'un conflit débouchant sur un procès interne. Elles deviennent alors un argument d'autorité, d'où l'importance du rôle du secrétaire et du cahier du Capitulo. La fonction de secrétaire est élective, renforçant le caractère démocratique de La Asociación. Si le secrétaire est important dans le quotidien de son groupe, son prestige reste secondaire au regard de celui du président, de l'éventuel vice-président et, surtout, du chef de discipline. Peu de noms de secrétaires sont restés dans l'histoire des Netas, alors que certains présidents, tels que le Padrino, ont une renommée internationale. Seul Daniel a su se faire connaître et reconnaître par son travail. Cité en exemple par le Padrino, Daniel est surnommé le « secrétaire de La Asociación » parce qu'il collectionne, depuis l'origine du groupe en Équateur, tout ce qui a trait aux Ñetas : articles de presse, règles de conduite, messages internes, lettres de détenus. Aujourd'hui encore, il entretient des relations épistolaires avec Bebo et avec certains Netas à Porto Rico ou en Europe, afin de parfaire sa connaissance de La Asociación. Selon le Padrino, Daniel a en sa possession sept livres, qu'il a recopiés à la main sur de petits carnets et qui reprennent l'histoire complète des Netas. Avant qu'il ne parte vivre en Espagne, le Padrino en avait fait une copie pour l'emporter avec lui. Cependant, une fois installé à Barcelone, il commença à vendre du crack et, se croyant sous surveillance policière, il préféra donner les livres à d'autres présidents de Capítulos avec lesquels il est aujourd'hui en conflit. Profitant de mon voyage à Guayaquil au printemps 2014, le Padrino me demande de rencontrer Daniel et de lui ramener ces livres. Il organise ainsi mon séjour pour que je puisse rencontrer certains des membres de son ancien Capitulo, et que je me charge de photocopier les sept livres que je dois lui ramener. 
Je rencontre Daniel près d'un centre commercial, où il travaille comme gardien de sécurité. Cela fait maintenant plusieurs années qu'il n'est plus affilié à un Capitulo en particulier. S'il se considère comme toujours actif, il ne participe plus aux activités quotidiennes d'un Capitulo, mais se concentre sur ses recherches sur La Asociación. Les sept livres dans un sac à dos, nous nous dirigeons vers une imprimerie pour pouvoir faire des photocopies. Plutôt que de livres, il s'agit de sept documents de 20 à 60 pages chaque, reliés par une spirale. Deux d'entre eux sont remplis d'articles de presse concernant les Netas à Guayaquil, deux autres contiennent l'histoire de La Asociación et les trois derniers sont composés de mensajes de conciencia, des lettres écrites par les membres et publiées au sein du Capitulo. Cette importance de l'écrit ne relève pas du seul travail de compilation de Daniel : depuis les années 1990, les Netas ont développé une pratique d'écriture qui a pris une place centrale dans leurs activités.

\section{Transformation interne et développement de l'écriture}

Quatre sortes d'écrits ponctuent la vie de La Asociación. La première concerne l'histoire que les Ñetas ont écrite d'eux-mêmes et la vie de leur héros fondateur, Carlos La Sombra. La deuxième concerne l'organisation interne des Netas, regroupant par exemple les règles et la discipline, ainsi que les punitions encourues par les membres qui transgressent ces règles. Le troisième type d'écrits est constitué des mensajes de conciencia (messages de conscience), les messages à teneur politique, personnelle ou spirituelle qui sont diffusés aux autres membres. Enfin, les lettres qui peuvent être adressées à une personne, un groupe de personnes ou à l'ensemble des Ñetas constituent le quatrième type d'écrits. Les trois premières catégories d'écrits - l'histoire, les règles et une partie de mensajes de conciencia -, sont regroupées dans un petit livre appelé en Espagne le guia liderato máximo (le guide du chef suprême) ou, plus communément, le Liderato. D'environ quatre-vingts pages, le Liderato comprend le récit de la vie de Carlos La Sombra, le fondateur de La Asociación, la philosophie des Ñetas, les règles internes, l'organigramme du groupe, les prières et le déroulé du rituel du Grito, cérémonie en l'honneur de Carlos La Sombra, ainsi que quelques messages de conscience. Les sept livres de Daniel contiennent le Liderato espagnol plus une série d'autres documents, tels que des articles de presse sur les Netas.

Cet effort de mise par écrit est intervenu au milieu des années 1990 à New York, alors que les Ñetas opéraient une transformation radicale de leurs activités. Cette réorganisation est, par ailleurs, contingente de celle d'autres gangs, comme les Latin Kings ou la Zulu Nation ${ }^{16}$. Au début des années 1990, les Netas entrent en effet en guerre pour le contrôle du territoire avec des gangs tels que les Latin Kings, la Zulu Nation ou encore la Familia. Les Netas ne sont pas encore organisés et centralisés, et plusieurs groupes naissent de 
manière relativement isolée les uns des autres. Les Ñetas, catégorisés comme gang, tant par les pouvoirs publics, les médias que par les membres euxmêmes, comprennent alors plusieurs milliers d'hommes et de femmes. La plupart des membres participent aussi activement au trafic de drogue et d'armes à feu, notamment à Harlem et dans le Bronx, ce qui renforce les guerres de territoire et les rend plus violentes ${ }^{17}$. Mais, à partir des années 1994-1995, les Netas organisent la première Junta Central, une super structure regroupant l'ensemble des sections locales à l'échelle de New York, appelée Tri State. Influencés par d'anciens activistes radicaux des années 1970, notamment par d'anciens membres du Young Lords Party, les présidents de la Junta impulsent un mouvement de "retour aux sources » et à l'histoire de La Asociación. En effet, l'histoire politique - pro-indépendantiste et socialiste - des Netas à Porto Rico a été en partie oubliée par les membres new yorkais lors de leur implantation dans le Bronx et à Brooklyn. Les Netas organisent alors des "pèlerinages » à Porto Rico, où ils se rendent sur la tombe de Carlos, parlent à sa famille ainsi qu'à d'anciens camarades du Parti Socialiste Portoricain auquel Carlos était affilié. Ils rédigent à leur retour les résultats de leur enquête dans ce qui deviendra le Liderato, le livre des Netas new yorkais.

À partir du milieu des années 1990, les Ñetas new yorkais commencent à organiser des réunions d'éducation informelle dans les caves, les parcs et les halls d'entrée, où ils apprennent l'histoire de Carlos et s'éduquent aux principes politiques des groupes comme les Young Lords : indépendantisme, anticolonialisme, anti-impérialisme, socialisme, démocratie directe et action collective. C'est à peu près au même moment que La Asociación s'étend à la côte Ouest de l'Amérique Latine, puis plus tard à l'Europe. Cette circulation permet la diffusion de l'histoire de Carlos. Celle-ci fut en effet retranscrite dans plusieurs petits carnets à Guayaquil, notamment par Daniel, avant d'arriver en Espagne.

Que ce soit aux États-Unis, en Amérique Latine ou en Europe, l'écriture a une place centrale dans La Asociación. Pour devenir Netas, le futur membre doit suivre un long processus d'initiation, appelé la convivencia, qui est fina-

16. Brotherton D. et L. Barrios, op. cit. ; sur la transformation des gangs, voir : Moore J., Going down to the Barrio: Homeboys and Homegirls in Change, Philadelphia, Temple University Press, 1991, p. 192 ; Hagedorn J., The In\$ane Chicago Way: The Daring Plan by Chicago Gangs to Create a Spanish Mafia, Chicago, Chicago University Press, 2015, p. 320; Densley J. A., How Gangs Work: An Ethnography of Youth Violence, Basingstoke, Hampshire, Palgrave Macmillan. 2016, p. 213 ; Decker S. et Pyrooz D.C., Moule R.,. « Disengagement From Gangs as Role Transitions ", Journal of Research on Adolescence, vol. 24, $\mathrm{n}^{\circ} 2,2014$, pp. 268-83 ; Ayling J., "Gang Change and Evolutionary Theory », Crime, Law and Social Change, vol. 56, n 1, 2011, pp. 1-26; Rodgers D., "Critique of Urban Violence: Bismarckian Transformations in Managua, Nicaragua », Theory, Culture E Society, vol. 33, n 7-8, 2016, pp. 85-109 ; Rodgers D. et J. Hazen, Global Gangs: Street Violence across the World, Minneapolis, University of Minnesota Press, 2014, p. 300.

17. Curtis R., «The Improbable Transformation of Inner-City Neighborhoods: Crime, Violence, Drugs, and Youth in the 1990s ", Journal of Criminal Law and Criminology, vol. 88, n 4, 1998, pp. 1233-1270. 
lisé lors d'une cérémonie où lui seront posées des questions sur l'histoire de Carlos, telles que sa date de naissance, le nom de ses enfants ou les dates de ses diverses arrestations. Pour apprendre cette histoire, le président du Chapter se sert des paperwork dont il distribue les pages concernant la vie de Carlos. Être Netas ne se résume plus à la participation aux guerres de gangs, mais devient lié à une histoire commune, celle de Carlos, qui est apprise et qui agit comme socle commun à la communauté. Pour autant, si la dimension anti-impérialiste ou socialiste des Ñetas à New York circule avec l'histoire de Carlos, elle est progressivement mise en arrière-plan au profit d'une philosophie de vie moins précise où prime un rejet de l'autorité de l'État, notamment de l'administration carcérale. De nombreuses références s'ajoutent à celles des Young Lords, de la lutte du peuple Mapuche, de Che Guevara, sans pour autant constituer une véritable idéologie politique articulée.

\section{Message de conscience}

Avant de nous séparer, Daniel me demande d'écrire un mensaje de conciencia qui sera distribué aux membres avant d'être archivé. Depuis un locutorio, un café internet, il me montre sur son compte email tous les messages d'autres membres qu'il a gardés. Ceux-ci restent en attente d'être archivés dans un autre livre qui s'ajoutera aux sept que j'emporte avec moi. Dans ces livres en effet, comme dans le liderato espagnol, sont mis bout à bout des messages que les membres adressent de manière publique à l'ensemble des Netas. Ces messages peuvent être des notes personnelles sur des situations endurées, des requêtes particulières, des plaintes générales suite à des événements récents ou des réflexions sur le rôle et la place des Netas. Ils sont un moyen de parler des peurs, des difficultés de la vie, mais aussi des transformations, du bien et du mal, de ce que La Asociación devrait être et de la manière de vivre une vie honnête. Écrits et signés par leurs auteurs, ces messages rejoignent les papiers archivés par Daniel ou par les présidents des Capitulos concernés. Ils peuvent être lus à l'oral lors d'une cérémonie ou d'une réunion. Tout Hermanito a le droit de prendre la parole et chacun incorpore un peu de lui-même dans les écrits du groupe en y inscrivant son message ${ }^{18}$. Ils deviennent alors un élément du texte, qui cimente à son tour le groupe. Puisque ces écrits sont diffusés aujourd'hui comme messages électroniques sur les réseaux sociaux, leur matérialité n'est pas la même que le Liderato qui est, lui, sous forme papier. Leur compilation est donc d'autant plus facile, de même que leur archivage, sur un disque dur ou dans une boîte email. Ces textes permettent de créer et recréer continuellement, par le biais de ces ajouts internes, une alliance fraternelle entre les membres. Pour reprendre la formule de Deborah Puccio Den sur le théâtre religieux en Espagne, il y a une forme de « consan-

18. Herrou A., "Quand les moines taoïstes en Chine "se mettent en texte" ", in Baptandier B. et G. Charuty, (eds.), Du corps au texte : approches comparatives, Nanterre, Société d'ethnologie, 2008, p. 351. 
guinité du texte 19 » se développant à l'échelle du Monde Netas, qui s'inscrit ici dans la circulation des écrits. Cette participation aux écrits permet de nuancer la dimension autoritaire de La Asociación et de la communication unidirectionnelle des lettres de Papi Caro. Le dialogue se fait ainsi à un niveau plus horizontal puisque chaque membre peut y prendre part. Les secrétaires des sections locales peuvent incorporer ces messages de conscience au cahier du Chapter, afin d'en garder une trace, et ces textes peuvent être discutés lors des réunions de Chapter. Cependant, ces messages de conscience n'ont pas, comme ce peut être le cas des lettres du líder máximo, une valeur juridique interne. Ils n'ont pas non plus une dimension sacrée, comme ce peut être le cas du Liderato, qui ne peut être annoté ou posé à même le sol. Ils permettent l'expression d'une voix personnelle et le déploiement d'un imaginaire propre. Par ailleurs, si chaque membre peut publier ses textes sur les réseaux sociaux, et prendre ainsi part aux discussions de la communauté Ñetas, seuls quelquesuns sont incorporés directement dans le Liderato qui circule en Espagne en version papier. Il s'agit des messages de conscience des personnalités Ñetas importantes, comme le chef de la Junta ou certains chefs de sections locales.

\section{Tournant bureaucratique et archives : le pouvoir du secrétaire}

Dans les années 1990, à l'époque où le Liderato est écrit, les Ñetas de New York ont centralisé le pouvoir au sein de la Junta afin de mieux contrôler les activités et l'organisation interne des Chapters. Cette centralisation s'est accompagnée, par ailleurs, d'un «tournant bureaucratique » de La Asociación, marqué par le recours systématique à l'écrit et un processus d'archivage des papiers. Le processus engagé à New York dans les années 1990 sera poursuivi en Équateur et en Espagne peu de temps après l'implantation des Ñetas dans ces deux pays.

La fonction de Daniel répond ainsi à une nécessité toute nouvelle dans l'histoire de La Asociación qui, en se centralisant, développe une véritable bureaucratie administrative et garde une trace écrite des affaires en cours et des décisions des présidents. L'archivage constitue un mode d'action dans le temps et un acte matériel venant en support de l'autorité des personnages qui, comme Daniel, continuent de peser dans les relations de pouvoir interne au Chapter. En contrôlant les papiers qui doivent être gardés ou non, en consignant les procès et les sanctions, en archivant certaines histoires plus que d'autres, les secrétaires ont un réel poids dans la vie quotidienne des Chapters et dans la direction empruntée par La Asociación.

Du fait de la petite taille du Capítulo dans lequel j'étais engagé, de sa relative jeunesse et, surtout, de l'autorité de son président, le Padrino, l'un des

19. Puccio Den D., «La Morisma en chair et en os. Pratiques et représentations autour d'un texte théâtral (Ainsa, Aragon) », in Baptandier B. et G. Charuty, op. cit., , pp. 75-110. 
Netas les plus expérimentés à Barcelone, mon rôle de secrétaire fut aussi modeste qu'effacé. Si les positions de président de section ou de chef de la discipline sont chèrement attribuées, et donc compétitives, celle de secrétaire reste une position de second ordre. Pour autant, ce rôle peut être exploité, comme le fit Daniel, afin d'acquérir reconnaissance et pouvoir au niveau local. Le cas de Bebo en est un autre exemple, puisque c'est à partir de la position de secrétaire de la Junta Central qu'il commença à accumuler prestige et pouvoir.

\section{Prévoir : les plans du président}

\section{Le plan de réunification mondiale}

En mai 2014, Mikey, président du Chapter de New York après l'éviction de Bebo, convie le Padrino ainsi que plusieurs présidents de Capitulos équatoriens et chiliens à une réunion virtuelle sur Internet. Lors de cette réunion, Mikey annonce l'intention des Netas new-yorkais de lancer un plan de travail au «niveau mondial ", qui viserait à terme à réunir l'ensemble des Netas. Depuis plusieurs années en effet, les Ñetas ne semblent plus réussir à se coordonner au niveau mondial, chaque Chapter organisant ses propres activités sans en référer à ceux des autres pays. De fait, l'expulsion de Bebo, qui maintenait un contact permanent entre les Netas dans le monde, a fait émerger des désaccords. N'étant pas convié à la réunion, le Padrino me raconte en détail la proposition du Chapter new yorkais. Selon Mikey, le plan qu'il propose a été approuvé par Papi Caro, le líder máximo et doit maintenant être appliqué. Ce plan, intitulé «Plan de trabajo mundial » (plan de travail mondial) a pour but, entre autres, de développer l'éducation des Capitulos sur la philosophie Netas et de poursuivre l'effort mis en place à New York de réduction des guerres de gangs.

La centralisation de La Asociación à New York dans les années 1990 a, en effet, eu pour conséquence la fin des guerres de gangs entre les Netas et les Latin Kings. La création d'une Junta Central supervisant les Chapters a permis un plus fort contrôle des membres à l'échelle de New York. La Junta a, ainsi, réduit le nombre de Chapters, qui passent de trente-trois en 1993 à treize en 1995 pour le South Bronx uniquement. Du fait de cette restructuration, à partir du milieu des années 1990, les membres de certains Chapters ne vivent plus dans la zone d'influence de ce dernier et ne prennent pas part aux activités du quartier. Cela a une implication directe sur les guerres de gangs, notamment dans les écoles. Bien qu'appartenant à un Chapter du South Bronx, les membres sont dispersés à l'échelle de la ville et ne vivent plus ces relations du quotidien qui caractérisaient la vie des Ñetas au début de leur implantation à New York. Une bagarre éclatant dans une école du Bronx entre un Latin King et un Netas a moins de chance de se propager en guerre de territoire, puisque la plupart des membres des Chapters concernés vivent dans d'autres quartiers 
de la ville et sont inscrits dans d'autres réseaux de socialisation. Ces guerres diminuent drastiquement alors que change peu à peu la raison d'être de $\mathrm{La}$ Asociación. Ce mouvement de déterritorialisation va de pair avec un processus de retour à l'histoire politique de La Asociación initié par les leaders de la Junta central. Au milieu des années 1990, les Ñetas passent ainsi des traités de paix avec leurs anciens ennemis : Latin Kings, Zulu, La Familia. Tous participent, d'ailleurs, ensemble aux grandes manifestations contre les violences policières en constituant le service d'ordre. Ce mouvement de pacification est accompagné et facilité par la mise en écriture de l'histoire de La Asociación, qui correspond à la nouvelle politique interne des Ñetas à New York.

Lors de cette première réunion virtuelle, le Padrino se dispute fermement avec Mikey sur plusieurs points. Ce dernier demande, par exemple, aux Ñetas de Barcelone de changer le nom d'un de leur Capitulo, car il est identique à celui d'un groupe ennemi des Netas à New York. Furieux, le Padrino m'explique :

Le Padrino : Et puis, tu te rends compte, ils veulent nous éduquer... Bon, en fait, ils veulent nous contrôler, quoi. Ils veulent écrire leur histoire et leur philosophie... En plus, ils disent que ceux qui n'acceptent pas le plan ne seront plus Ñetas, et que c'est Papi Caro qui l'a dit. En passant par Porto Rico, il essaie de prendre le pouvoir. Mais bon, nous, on s'en fout de Porto Rico... C'est eux qui ont dit qu'on n'était pas de vrais Netas. Entre temps, je discutais par Internet avec ceux d'Équateur et ceux du Chili de mon plan... et on rigolait bien. Du coup, nous, on va faire notre plan. Et si Mikey veut pas de nous, il sera tout seul. De toute façon, maintenant, les Ñetas, c'est $90 \%$ des Équatoriens 20.

La dispute se poursuit lors des sessions de travail suivantes, et confirme le désir du Padrino d'établir son propre plan, qu'il appelle «Plan de reunificación mundial ", le plan de réunification mondiale. Le Padrino veut, en effet, réunir les Netas de Barcelone et ceux de Madrid dans une Junta Central à l'échelle de l'Espagne. Il envisage, ensuite, de rétablir les contacts avec les Chapters en Équateur et au Chili. La Junta Central à Barcelone et la fin des guerres de gangs dans la ville catalane avaient, en effet, créé une certaine tension entre Netas en Espagne et en Amérique Latine. Le Padrino, autrefois connu pour être un président cruel et sanguinaire à Guayaquil, a été l'un des artisans de la paix à Barcelone et cette nouvelle position fut mal perçue à Guayaquil, où les guerres de gangs ont toujours cours, bien que de manière moins virulente qu'au début des années 2000. Ne comprenant pas ce revirement, certains Netas équatoriens qualifièrent à ce moment le Padrino de trấtre.

20. Note de la conversation a posteriori sur mon carnet de terrain. 
Ainsi, lorsque j'annonce pour la première fois au Padrino mon souhait d'aller en Équateur, ce dernier tente de m'en dissuader. Guayaquil est une ville violente où il ne peut pas assurer ma sécurité. Cependant, ce voyage entre dans ses plans de réunification et il accepte de m'aider dans ma démarche. Profitant de mon voyage, il me demande d'apporter des messages à certains Ñetas importants en Équateur. Depuis son compte Facebook, le Padrino est en pourparlers avec les Netas du Chili, de Guayaquil et Bebo, afin de centraliser les initiatives et de coordonner le mouvement. Pour donner plus de poids à son projet, il veut expliquer aux membres de Guayaquil le type de travail qu'il entreprend à Barcelone et il me charge d'apporter des messages écrits officiels et privés. Chacune des lettres contient des salutations respectueuses, une explication de ma présence à Guayaquil et du projet du Padrino. Elles présentent, entre autres, le désir de réunification du Padrino, et demandent à chaque président de Capitulo auquel je les transmets, de bien vouloir participer au projet. Si le Padrino n'a pas besoin de lettres écrites pour être en dialogue avec ces derniers, celles-ci donnent un ton plus officiel à sa démarche. En effet, chaque partie gardera la preuve de cet échange dans les archives des Capitulos. La circulation de ces écrits permet au Padrino de rétablir un lien formel entre l'Espagne et l'Équateur, lien qui ne passe plus ni par New York, ni par Porto Rico. Par ailleurs, il est stratégiquement plus intéressant pour lui de m'envoyer comme messager, plutôt que de passer par les réseaux sociaux. D’une part, cela prouve la capacité logistique et la puissance de son Capitulo, en mesure de faire voyager son secrétaire de l'autre côté de l'Atlantique. D'autre part, cela montre le sérieux du projet et le respect que le Padrino accorde à ses interlocuteurs. En arrivant à Guayaquil, je me trouve ainsi à mes dépens - tout en profitant largement du type d'accès que cette position me donne - porteur des lettres du Padrino 21. Que ce soit les lettres ou les sept livres, ces écrits auraient facilement pu circuler via Internet, ce qui atteste de l'importance du papier dans les démarches officielles au sein de La Asociación.

\section{Régimes d'efficacité du plan}

Les plans de travail sont courants dans La Asociación. Durant mes quatre années de terrain, j'ai pu voir une dizaine de projets de plan se mettre en œuvre, mais surtout échouer. Qu'il s'agisse du plan de réunification à l'échelle mondiale ou locale, de plans pour organiser des séances de discussions ou des manifestations, les Ñetas passent un temps important à établir des projets, leur donner une forme écrite, en discuter lors des réunions de Chapters, ou de réunions entre présidents. Une partie de l'activité de ces derniers réside, d'ailleurs, dans l'organisation de plans. Ceux-ci varient en degré d'implication,

21. Avant de devenir messager, j'ai posé comme condition de pouvoir lire les textes que j'allais apporter, en promettant toutefois de ne pas révéler la teneur des messages dans mes publications. J'ai, par ailleurs, systématiquement refusé de transporter des sacs ou autres marchandises. Je peux ainsi assurer que ces textes ne contenaient rien d'illégal. 
d'échelle et de temps. Par exemple, un des Capítulos proche de celui du Padrino mettra en place un plan de tournoi de foot entre plusieurs Capitulos afin de récolter de l'argent pour un membre incarcéré. Dans la même aprèsmidi, alors que la plupart des membres participent aux matchs, les présidents des Capitulos invités se retrouvent pour discuter du plan du Padrino. En 20142015, le Padrino effectue plusieurs voyages à Madrid pour discuter de son plan de réunification mondiale avec certains Capitulos de la ville. Il passe aussi beaucoup de temps au téléphone avec Bebo afin de lui exposer sa stratégie et le convaincre d'impliquer le Chapter du New Jersey.

Mis à part les plans très locaux, comme le tournoi de foot à Barcelone ou la participation à une manifestation à New York, les plans d'envergure plus internationale, que ce soit le Plan de trabajo mundial ou le Plan de reunificación Mundial, restent assez peu concrets. Il s'agit dans les deux cas de rétablir les contacts et d'œuvrer à l'établissement d'une vision somme toute assez similaire : l'arrêt des guerres de gangs. Aucune activité n'est organisée et le plan consiste en la programmation d'une série de discussions. De fait, aucun des deux plans n'aboutira, qu'il avorte pour cause de conflits dans le premier cas, ou soit suspendu pour raisons personnelles dans le second.

Pour autant, il serait erroné de penser que, parce qu'ils échouent à se mettre en œuvre, ces plans n'ont pas une certaine efficacité. Au contraire, la plusvalue de l'organisation du plan n'est pas dans le plan lui-même, mais dans la démarche d'organisation. Comme l'indique Yves Cohen, le plan est un instrument et un support de l'autorité du chef. Il permet de prouver la capacité du Padrino à s'organiser et, donc, à être un chef. En étant à l'initiative, le Padrino signe le projet, même si son nom n'est pas apposé en bas de pages. L'établissement de ce plan est en soi une action d'autorité qui fixe le cadre de l'action. Le plan est une forme de gouvernement pour le Padrino. Par ailleurs, son organisation et la prise de contact avec divers présidents de Capítulos en Amérique Latine et en Espagne renforcent la notoriété du Padrino dans le Monde Ñetas, ainsi que son autorité dans son propre Capitulo. Il s'impose comme un président d'action, capable de développer des stratégies et fort d'un réseau international imposant. Enfin, une partie de ce plan consiste à comploter à l'encontre du Chapter de New York et de son président Mikey, ce qui resserre les liens entre Bebo et le Padrino. Ce dernier réussira, d'ailleurs, à obtenir de la part de Bebo le téléphone de Papi Caro et à entrer en contact à plusieurs reprises directement avec lui. Les liens téléphoniques privilégiés avec le líder máximo répondent à plusieurs stratégies. Pour le Padrino, il s'agit à la fois de prouver aux Ñetas son appartenance à un petit groupe de privilégiés et de rendre son projet au moins aussi important que celui de Mikey. Pour le líder máximo, il s'agit d'avoir des contacts au-delà de l'espace de sa cellule. Cette stratégie permet de développer ce que Yves Cohen appelle un système de primes visant, pour le líder máximo à s'attacher durablement des yeux et 
des oreilles dans un monde qu'il dirige mais ne peut arpenter. Enfin, pour Bebo, il s'agit de retrouver une place dont il a été exclu et de pouvoir peser de nouveau dans les relations entre Netas, à New York comme à Porto Rico.

\section{Trois logiques d'autorité et d'écriture chez les Ñetas}

Ces trois exemples de mobilisation d'écrits - lettres, archivage, plans montrent la diversité des modalités d'action qui constituent autant de sources d'autorité.

Tout d'abord, les échanges épistolaires entre le líder máximo et le reste du Monde Netas sont un instrument d'autorité à distance pour le chef suprême. Ces lettres, en se diffusant, relaient l'autorité du chef, infiltrant de "manière capillaire 22 »le Monde Ñetas et intensifiant la présence d'un chef pourtant distant. Mais si les lettres transportent l'autorité du chef, elles rendent aussi atteignable le Monde Ñetas au chef ou, autrement dit, elles mettent le monde à la portée du líder máximo, emprisonné dans sa cellule. La globalisation des Netas oblige ce dernier à développer des techniques de communication et de gouvernement, faisant de sa distance le cœur de son autorité, presque sacrale.

Pour autant, ce principe centralisateur d'autorité est contrebalancé par un rapport à l'écrit diffus et horizontal, ce que montre l'exemple de Daniel. L'archivage des textes permet de garder une trace précise des affaires passées alors que les mensajes de conciencia autorisent chaque membre à s'emparer de l'écrit et à faire partie du texte. C'est un argument d'autorité qui répond, par ailleurs, au besoin d'une bureaucratie concomitante de la centralisation des Capitulos en une Junta Central. Mais l'archivage constitue aussi un acte matériel venant en support de l'autorité de celui qui garde trace des écrits.

Enfin, la constitution d'un plan et son écriture sont instruments et support de l'autorité du président, au niveau de son Capitulo, mais aussi à l'échelle du Monde Ñetas. À la différence des mensajes de conciencia, le plan n'est pas signé par son auteur. Mais il est en lui-même empreint de la vision de ce dernier qui, si le plan est accepté, dirigera les actions futures de la collectivité entière. L'établissement du plan procède d'un acte d'autorité individuelle. Bien qu'ils ne soient pas menés à terme, ces plans mobilisent la communauté Netas et le projet du Padrino lui permet de renouer des contacts et de contourner les Ñetas New Yorkais, initiant une nouvelle polarité dans le monde Netas. Les lettres que le Padrino envoie aux Netas équatoriens constituent ainsi un support du Monde Neta, dessinant par leur circulation le nouveau contour et les nouvelles routes de ce monde, ses forces et ses polarités. Ici, ce sont d'autres vecteurs relationnels qui se développent, quelque peu en dehors du lien avec Porto Rico et, surtout, New York.

22. Puccio Den D., op. cit., 2014. 
Yves Cohen nous invite justement à décliner les actions du chef selon l'espace et à penser ses actes en termes d'actes spatiaux. C'est donc aussi une pragmatique des actes spatiaux que dévoile l'existence de ces échanges pluriels et qui consolide le Monde Ñetas. Ainsi, par exemple, l'espace du chef portoricain est particulier, à la fois confiné à sa cellule et à la dimension du Monde Netas. Par leur circulation, les lettres rendent matérielles à la fois la présence du chef et le monde qu'elles dessinent. Les lettres se font, alors, véhicules de l'imagination du chef et du Monde Netas pour ceux qui font partie de la correspondance. La présence du chef est en même temps un mode de connaissance, explique Yves Cohen - et donc de reconnaissance du Monde et de son secret - et une technique de surveillance. Enfin, la présence spectrale du líder máximo se matérialise par le document même, autant qu'à travers ses procédures de contrôle.

La circulation de ces lettres, messages de conscience et plans me permet de retracer le contour du Monde Netas qui se déplie alors et est mis en action, en mouvement. Pour cela, il s'agit de suivre la diversité des routes de circulation entre les villes. Ces divers écrits permettent de faire tourner le Monde Ñetas, mais aussi de le faire tenir.

Dans cette attention portée à ce qui circule, encore faut-il ne pas oublier les différentes forces et pouvoirs que ces écrits exercent. Les échanges épistolaires avec Papi Caro permettent de mettre à jour une configuration des relations de prestige entre les Hermanitos (frères Netas) dont les positions sont définies par le type d'échanges (téléphoniques, écrits) avec le líder máximo ${ }^{23}$. Mais le passage en force du plan du Padrino sur celui de Mikey éclaire une redistribution des hiérarchies et des polarités dans le Monde Ñetas. Il est ainsi possible de cartographier à la fois le mouvement des actants ${ }^{24}$ et de décrire les forces de gravitation impliquées ${ }^{25}$. Le Monde Netas est fait de hiérarchies et de polarités où tous les nœuds n'ont pas la même force ou le même niveau. Il faut donc penser de manière processuelle le réseau et le territoire, la territorialité du réseau et la réticularité du territoire.

L'écriture, selon ses supports et ses usages, a plusieurs fonctions chez les Netas. Elle peut avoir un caractère dépositaire de l'histoire, lorsqu'il s'agit du Liderato, le livre des Netas. Mais elle peut aussi avoir un caractère de

23. Déborah Puccio-Den notait déjà une reconfiguration des relations entre hommes d'honneur de la Cosa nostra en fonction de leur rang dans les échanges épistolaires avec Provenzano. Puccio-Den D., op. cit., 2014. L'auteure cite par ailleurs J. Goody sur la redistribution des hiérarchies produite par l'irruption de l'écriture dans des sociétés à tradition orale. Goody J., La raison graphique : la domestication de la pensée sauvage, Paris, Éditions de Minuit, 1979, p. 272.

24. Bertrand R., L'histoire à parts égales: récits d'une rencontre Orient-Occident, (XVIe-XVII siècle), 2014, p. 500.

25. Anderson B., Les bannières de la révolte: anarchisme, littérature et imaginaire anticolonial: la naissance d'une autre mondialisation, Paris, La Découverte, 2009, p. 260. 
construction collective, lorsqu'il s'agit des messages de conscience qui, en circulant, fondent et identifient ce qui fait communauté. Mais ces trois types de prises d'écriture sont soutenus par trois logiques d'action correspondant à trois rôles différents que j'ai désignés dans cet article : diriger pour le líder máximo, ordonnancer pour le secrétaire et prévoir pour le président local. Les enjeux pour ces trois figures sont différents. Pour le líder máximo, l'écriture est une technique de management lui permettant de contrôler le Monde Ñetas et d'y être présent. L'échelle qu'il mobilise doit être la plus grande possible alors que son espace d'action immédiat est réduit et contenu. Pour ce faire, il a besoin de maintenir un espace des effets le plus vaste possible. Le secrétaire, au contraire, a une action réduite en termes d'échelle de zone d'activité. Par contre, son action est grande, ou plutôt dense, à la fois en termes de contrôle du quotidien et de la vie des membres, ainsi que du rapport à l'histoire de $\mathrm{La}$ Asociación. Enfin, le président de Capitulo cherche à augmenter sa zone d'activité et l'espace de son action immédiate tout en ayant une zone d'impact importante. Mais il y a aussi une certaine densité temporelle à ses logiques d'action puisqu'un président doit engager l'avenir de ses membres, qu'il s'agisse de s'associer avec d'autres membres ou d'entrer en guerre. Pour cela, il utilise le plan comme «mode d'action sur l'avenir » lui permettant de prévoir et «d'agir dans et sur le temps 26 ».

26. Cohen Y., op. cit., p. 487. 